\title{
Phase II study of S-1 and docetaxel for previously treated patients with locally advanced or metastatic non-small cell lung cancer.
}

\section{AUTHOR(S):}

Yanagihara, Kazuhiro; Yoshimura, Kenichi; Niimi, Miyuki; Yasuda, Hiroyasu; Sasaki, Takahiko; Nishimura, Takafumi; Ishiguro, Hiroshi; ... Teramukai, Satoshi; Mio, Tadashi; Fukushima, Masanori

\section{CITATION:}

Yanagihara, Kazuhiro ...[et al]. Phase II study of S-1 and docetaxel for previously treated patients with locally advanced or metastatic non-small cell lung cancer.. Cancer chemotherapy and pharmacology 2010, 66(5): 913-918

\section{ISSUE DATE:}

2010-10

\section{URL:}

http://hdl.handle.net/2433/129639

\section{RIGHT:}

The original publication is available at www.springerlink.com; この論文 は出版社版でありません。引用の際には出版社版をご確認ご利用くだ さい。; This is not the published version. Please cite only the published version. 


\section{ORIGINAL ARTICLE}

Phase II study of S-1 and docetaxel for previously treated patients with locally advanced or metastatic non-small cell lung cancer

Kazuhiro Yanagihara ${ }^{1,2^{\dagger}}$, Kenichi Yoshimura ${ }^{3^{\dagger}}$, Miyuki Niimi $^{3}{ }^{\dagger}$, Hiroyasu Yasuda ${ }^{1}$, Takahiko Sasaki $^{1}$, Takafumi Nishimura ${ }^{1,2}$, Hiroshi Ishiguro ${ }^{1,2}$, Shigemi Matsumoto ${ }^{1,2}$, Toshiyuki Kitano ${ }^{1,2}$, Masashi Kanai ${ }^{1}$, Akiko Misawa ${ }^{1}$, Harue Tada $^{3}$, Satoshi Teramukai ${ }^{3}$, Tadashi Mio ${ }^{4}$, Masanori Fukushima ${ }^{1,3}$

${ }^{\dagger} \mathrm{KY}, \mathrm{KY}$ and MN equally contributed to this work.

Affiliation:

1 Outpatient Oncology Unit, Kyoto University Hospital, Kyoto, Japan

2 Department of Translational Clinical Oncology, Graduate School of Medicine, Kyoto University, Kyoto, Japan.

3 Department of Clinical Trial Design and Management, Translational Research Center, Kyoto University Hospital, Kyoto, Japan

4 Department of Multidisciplinary Cancer Treatment, Graduate School of Medicine, 
Kyoto University, Kyoto, Japan

Correspondence: Kazuhiro Yanagihara, M.D., Ph.D.

Department of Translational Clinical Oncology,

Graduate School of Medicine, Kyoto University

54 Shogoin Kawahara-cho, Sakyo-ku, Kyoto, 606-8507, Japan

Phone: $+81-75-751-4770$

Fax: +81-75-751-4772

E-mail: kazuhiro@kuhp.kyoto-u.ac.jp 


\section{Abstract}

Purpose The purpose of the present phase II study was to evaluate both the efficacy and toxicity of the combination of S-1 and docetaxel in previously treated patients with locally advanced or metastatic non-small cell lung cancer.

Methods Thirty-eight previously treated patients with non-small cell lung cancer were treated with $\mathrm{S}-1\left(80 \mathrm{mg} / \mathrm{m}^{2}\right.$, days $1-14$, oral $)$ and docetaxel $\left(40 \mathrm{mg} / \mathrm{m}^{2}\right.$, day 1 , intravenous) every 3 weeks.

Results No complete response was observed, and seven patients had a partial response, yielding an overall response rate of $18.4 \%$ (95\% CI, $7.7-34.3 \%)$. The median overall survival time and 1-year overall survival rate were 16.1 months and $60 \%$, respectively. The median progression-free survival time was 4.4 months. Myelosuppression was the main toxicity with grade 3 or 4 neutropenia and leukopenia in $50 \%$ and $21 \%$, respectively. There was no irreversible toxicity in this study.

Conclusions The combination of S-1 and docetaxel is well tolerable and has substantial activity for patients with locally advanced or metastatic non-small cell lung cancer. A phase III trial comparing docetaxel with or without S-1 would warrant further investigation. 


\section{Key words}

non-small cell lung cancer, phase II study, docetaxel, S-1, second-line chemotherapy, third-line chemotherapy 


\section{Introduction}

Non-small cell lung cancer (NSCLC) is a leading cause of cancer deaths worldwide, but only a minority of patients is amenable to surgical or definitive chemoradiotherapy. The overall prognosis of NSCLC patients remains poor; only $15.2 \%$ patients are alive after 5 years [1]. Almost all patients eventually experience progression during or after treatment. Second-line chemotherapy with docetaxel showed modest antitumor activity, with overall response rate (ORR) of 6.7-7.1\%, and can prolong survival after failure of platinum-based regimens for NSCLC, with a 1-year overall survival (OS) rate of $21-31 \%$ [2, 3]. However, despite current evidences supporting the use of second-line chemotherapy, the modest survival benefits, the negligible low response rate and relevant toxicity may reduce the role of second-line chemotherapy in clinical settings.

S-1 (Taiho Pharmaceutical Co., Ltd.,, Tokyo, Japan) is an oral fluoropyrimidine agent comprising the 5-fluorouracil (5-FU) prodrug tegafur and two enzyme inhibitors, 5-chloro-2,4-dihydroxypyrimidine (CDHP) and potassium oxonate (OXO), in a molar ratio of 1:0.4:1. CDHP enhances the serum 5 -FU concentration by competitive inhibition of dihydropyrimidine dehydrogenase (DPD), an enzyme responsible for 5-FU catabolism. OXO is a reversible competitive inhibitor of orotate phosphoribosyl transferase (OPRT), 
a phosphoenzyme for 5-FU and reduces the gastrointestinal toxicity of 5-FU [4]. These mechanisms mean that oral S-1 administration can generate a higher concentration of 5-FU than protracted intravenous injection of 5-FU alone, while the incidence of toxicity in the gastrointestinal tract does not increase.

The combination of S-1 and docetaxel holds particularly great promise because both drugs have substantial antitumor activity as single agents, and they have different mechanisms of action and different toxicity profiles [2, 3, 5-7]. Recent preclinical studies have shown that S-1 has synergistic effects in human cancer xenografts [8-10]. The low level of DPD, thymidylate synthase activities, and a high level of OPRT activity enhance the antitumor effect of $5-\mathrm{FU}$ and $\mathrm{S}-1$. Docetaxel is one of the agents that modulate these enzyme expressions and activities. A phase I/II study has shown that this combination was well tolerated with moderate toxicities and promising activity in patients with gastric cancer [11]. Therefore, we conducted a phase II study to evaluate both the efficacy and toxicity of S-1 combined with docetaxel in previously treated patients with locally advanced or metastatic NSCLC.

\section{Materials and methods}


Eligibility criteria

Eligible patients were aged 20-74 years and had histologically or cytologically

confirmed locally advanced or metastatic NSCLC (stages IIIB-IV or relapse after surgery) that progressed after first- or second-line chemotherapy or chemoradiotherapy.

The patients were required to have measurable disease by the Response Evaluation Criteria in Solid Tumors (RECIST), an Eastern Cooperative Oncology Group (ECOG) performance status (PS) of 0 or 1, ability to take oral medication and normal ECG. Eligibility requirements also included a white blood cell count of $\leq 12,000$ cells $/ \mathrm{mL}$, an absolute neutrophil count of $\geq 2000$ cells $/ \mathrm{mL}$, a platelet count of $\geq 100,000$ cells $/ \mathrm{mL}$, a hemoglobin level of $\geq 9 \mathrm{~g} / \mathrm{dL}$, a serum total bilirubin level of $\leq 1.5 \mathrm{mg} / \mathrm{dL}$, a serum aspartate aminotransferase (AST)/alanine aminotransferase (ALT) of less than or equal to twice the upper limit of normal, a serum creatinine level of $\leq 1.5 \mathrm{mg} / \mathrm{dL}$ and a normal electrocardiogram. Prior thoracic radiotherapy was allowed as long as it had been completed at least 12 weeks prior to inclusion and the patient had recovered from any toxicity. At least 4 weeks had to have elapsed from prior surgery and completion of prior chemotherapy or chemoradiotherapy. Patients who had exhibited evidence of severe heart or pulmonary disease or concomitant malignancy were excluded. The protocol was approved by the Ethics Committee of Kyoto University, and every patient gave written 
informed consent. This trial was registered at University hospital Medical Information

Network, Japan (protocol ID number, UMIN000000501 at http://www.umin.ac.jp/).

Treatment plan

S-1 was given orally twice daily for 2 weeks, followed by a drug-free interval of 1 week (one cycle). Dose of S-1 administered each time was calculated according to the patient's body surface area as follows: less than $1.25 \mathrm{~m}^{2}, 40 \mathrm{mg} ; 1.25-1.5 \mathrm{~m}^{2}, 50 \mathrm{mg}$; and greater than $1.5 \mathrm{~m}^{2}, 60 \mathrm{mg}$. Docetaxel intravenous infusion $\left(40 \mathrm{mg} / \mathrm{m}^{2}\right)$ was administered on day 1 . The treatment regimen was repeated every 3 weeks until disease progression or intolerable toxicity occurred. For patients who experienced febrile neutropenia, hemorrhage with grade 3 or 4 thrombocytopenia, or grade 3 or 4 non-hematologic toxicity, the dose of docetaxel was to be reduced to $35 \mathrm{mg} / \mathrm{m}^{2}$ and the dose of $\mathrm{S}-1$ was also to be reduced to $80 \%$ of the initial dose. For patients who still experienced the same toxicity after the dose reduction, $\mathrm{S}-1$ was to be reduced to $80 \%$ of the reduced dose, and this could be done up to twice. If recovery from such toxicities at a reduced dose was confirmed, administration at the reduced dose was continued. Patients who still experienced the same toxicity after the dose reduction were to be withdrawn from the study treatment. 
Evaluation of response and toxicity

Patients underwent tumor assessments at baseline and every 6 weeks by investigators using RECIST. Patient survival was observed until death, loss to follow up, or study closure. Adverse events were recorded using the National Cancer Institute Common Terminology Criteria for Adverse Events (version 3).

Statistical analysis

The primary end point was the ORR as assessed in all eligible and treated patients, with success being defined as a complete response (CR) or partial response (PR) according to RECIST. The secondary endpoints were OS, progression-free survival (PFS) and adverse events. The design of this study was based on a binomial distribution with no planned interim analysis. Assuming a null hypothesis of a 9\% ORR and an alternative hypothesis of a $25 \%$ ORR, with one-sided type I error $=0.1$ and type II error $=0.1$, it was necessary to enroll a minimum of 35 patients. According to this, we aimed for 40 patients to take non-evaluable patients into consideration.

Exact confidence interval (CI) and exact P-value for ORR were based on the binomial distribution. OS was calculated from the date of registration until death from 
any cause, whereas PFS until disease progression or death from any cause. OS and PFS were analyzed using the Kaplan-Meier method. All statistical tests were one-sided, and a P-value of less than 0.05 was considered statistically significant. All analyses were performed using SAS version 9.1.3 (SAS Institute, Cary, NC, USA).

\section{Results}

Patient characteristics

Between August 2006 and December 2007, 42 patients were enrolled in this study according to the eligibility criteria. Thirty-nine of these 42 patients were eligible, of the remainder one patient had stage IIIA NSCLC and two patients were without adequate liver function. Following the study protocol, one eligible but untreated patient was excluded from the analysis because of the incidence of a compression fracture caused by osteoporosis before treatment. Baseline characteristics of the 38 patients are summarized in Table 1 . The median age was 65 years (range, 44-74 years). The majority of patients had an ECOG PS of 0 (95\%), had been histologically or cytologically diagnosed as having adenocarcinoma $(79 \%)$ and had progressed after at least one previous platinum-based chemotherapy regimen (92\%). The median number of courses administered per patient was five (range, 1-8). The median follow-up time was 17.2 
months.

Efficacy

Tumor response results are shown in Table 2. Among all treated patients, no CR was observed and seven patients had a PR, yielding an ORR of $18.4 \%$ (95\% CI, 7.7-34.3\%; $\mathrm{P}=0.05$ under the null hypothesis of a $9 \%$ ORR). Among the patients with adenocarcinoma, PR was observed in 4/30 (13.3\%). As shown in Figure 1, the median OS time was 16.1 months and the 1-year OS rate was $60 \%(95 \% \mathrm{CI}, 42.5-73.6 \%)$. The 1-year OS rates in stage-IIIB patients, stage-IV patients and patients with relapse after surgery were 70, 42 and 80\%, respectively. The median PFS time was 4.4 months, and the 1-year PFS rate was 37\% (Figure 1).

Safety

The major adverse events are shown in Table 3. The most frequent hematological toxicity was neutropenia with grade 3 or 4 neutropenia observed in $50 \%$ of patients. Of these events, grade 4 neutropenia was observed in seven patients (18\%) and febrile neutropenia in one patient (3\%). Grade 3 or 4 leukopenia was reported in $21 \%$ of patients. The non-hematological grade 3 toxicities were anorexia in five patients 
(13\%), stomatitis in four patients (11\%), hand-foot skin syndrome in two patients (5\%), diarrhea in two patients (5\%) and vomiting in one patient (3\%). There was no death or irreversible toxicity in this study that was considered to be related to treatment.

\section{Discussion}

Almost all patients with advanced NSCLC treated with first-line chemotherapy experience progression, and current options for the second-line treatment of NSCLC include single-agent chemotherapy with docetaxel, pemetrexed or erlotinib [12], which large-scale randomized clinical trials indicate as the standard regimen. However, the clinical responses to these agents are of short duration, and the survival benefit is limited.

Many reports have been published investigating combination chemotherapy using two non-platinum agents for recurrent NSCLC in randomized clinical trials, with the objective of improving outcomes further. However, none of these studies have demonstrated improved survival with combination chemotherapy, whereas there have been relatively higher or intolerable toxicities [13-16]. Therefore, more active regimens 
for the second-line chemotherapy are much needed.

In the present study, we evaluated the efficacy and safety of the combination of S-1 and docetaxel, two agents that separately have shown promise in the treatment of advanced or metastatic NSCLC. This combination chemotherapy conferred efficacy with an ORR of $18 \%$, a median OS time of 16 months and a 1 -year OS rate of $60 \%$. The $18 \%$ ORR observed in this study was slightly lower than expected. However, the survival benefits as second- or third-line therapy observed compare favorably with other chemotherapy regimens, such as monotherapy with docetaxel (6-14 months) $[2,3,7]$, pemetrexed (8 months) [17], erlotinib (6-15 months) [18-20] or oral topotecan (6-8 months) [21, 22], or combination chemotherapy of irinotecan and cisplatin (11 months) [23], or oral fluoropyrimidine UFUR and gemcitabine (13 months) [24], although between-study comparisons should be made with caution.

Prolonged survival may be due to substantial post-study treatment, especially epidermal growth factor receptor (EGFR) tyrosine kinase inhibitors (TKIs). Although EGFR mutation status was not analyzed in this study, 17 patients received EGFR-TKIs and 9 of those patients for over a month.

The hematological toxicity observed here was minimal and tolerable, despite the fact that grade 3 or 4 neutropenia occurred in 50\%, which is comparable with the 
toxicity caused by docetaxel monotherapy. The majority of non-hematologic toxicities were mild and tolerable without grade 4 non-hematologic toxicity. These toxicity results are consistent with those observed in a phase I/II study in patients with gastric cancer [11].

During the preparation of this manuscript, Atagi et al. [25] reported the results of a phase I/II study, in which the combination of S-1 and docetaxel was evaluated for patients who had failed one or more prior chemotherapy regimens. In the phase II part of their study, seven of 29 eligible patients achieved a PR, yielding an ORR of $24 \%$, with a median OS time and the 1-year OS rate of 12 months and $42 \%$, respectively. Patient characteristics were similar except for stage and ECOG PS: fewer patients who had experienced relapse after surgery were included, and 31\% and 69\% patients had ECOG PS of 0 and 1, respectively, in the study by Atagi et al. [25]. Although these differences in patient characteristics may lead to more favorable survival results in our study, the combination of S-1 and docetaxel still seems to be consistently promising as a chemotherapy option after the failure of prior chemotherapy for advanced NSCLC.

In this study, the dose of docetaxel was lower than that commonly used in docetaxel monotherapy. As a second-line docetaxel monotherapy, a dose of $75 \mathrm{mg} / \mathrm{m}^{2}$ every 3 weeks is used in the United States and Europe, and the dose is $60 \mathrm{mg} / \mathrm{m}^{2}$ every 3 
weeks in Japan. However, our regimen is widely recognized as a tolerable and optimized combination of S-1 and docetaxel in gastric cancer [11], and thus, also in lung cancer, it is considered promising in terms of toxicity and efficacy. Furthermore, it was the recommended dose in the phase I part of study reported by Atagi et al. [25].

There are many report of ethnic differences in the safety and efficacy profile of S-1 and docetaxel [25-28], and it is shown that CYP2A6*9 genetic polymorphism is a potential predictive marker, for efficacy and toxicity, for the patients received the combination of S-1 and docetaxel for metastatic gastric carcinoma [29]. In the development of a S-1/docetaxel combination therapy in the United States and Europe, further optimization of the dose of each agent may be required to account for these differences.

In conclusion, the combination of $\mathrm{S}-1$ and docetaxel is well tolerable and promisingly effective for patients with locally advanced or metastatic NSCLC. A phase III trial comparing docetaxel with or without $S-1$ would warrant further investigation.

\section{Acknowledgements}

Department of Translational Clinical Oncology, Graduate School of Medicine, Kyoto University was founded by the donation from Taiho Pharmaceutical Co., Ltd., Tokyo. 
Notes

$\mathrm{KY}, \mathrm{KY}$ and $\mathrm{MN}$ equally contributed to this work.

\section{References}

1. National Cancer Institute: Surveillance, Epidemiology, and End Results (SEER) Program. SEER Stat Database, National Cancer Institute, Surveillance Research Program, Cancer Statistics Branch. http://www.seer.cancer.gov/ Accessed 3 March 2009.

2. Shepherd FA, Dancey J, Ramlau R, et al. (2000) A prospective randomized trial of docetaxel versus best supportive care in patients with non-small-cell lung cancer previously treated with platinum-based chemotherapy. J Clin Oncol 18: 2095-2103.

3. Fossella FV, DeVore R, Kerr RN, et al. (2000) Randomized phase III trial of docetaxel versus vinorelbine or ifosfamide in patients with advanced non-small-cell lung cancer previously treated with platinum-containing chemotherapy regimens. J Clin Oncol 18: 2354-2362.

4. Shirasaka T, Shimamoto Y, Fukushima M (1993) Inhibition by oxonic acid of 
gastrointestinal toxicity of 5-fluorouracil without loss of its antitumor activity in rats. Cancer Res 53: 4004-9.

5 Furuse K, Kawahara M, Hasegawa K et al. (2001) Early phase II study of S-1, a new oral fluoropyrimidine, for advanced non-small-cell lung cancer. Int J Clin Oncol 6: 236-241.

6 Kubota K, Kawahara M, Ogawara M et al. (2008) Vinorelbine plus gemcitabine followed by docetaxel versus carboplatin plus paclitaxel in patients with advanced non-small-cell lung cancer: a randomised, open-label, phase III study. Lancet Oncol 9: 1135-42.

7 Maruyama R, Nishiwaki Y, Tamura T et al. (2008) Phase III Study, V-15-32, of gefitinib versus docetaxel in Previously Treated Japanese Patients With Non-Small-Cell Lung Cancer. J Clin Oncol 26: 4244-4252.

8 Wada Y, Yoshida K, Suzuki T, et al. (2006) Synergistic effects of docetaxel and S-1 by modulating the expression of metabolic enzymes of 5-fluorouracil in human gastric cancer cell lines. Int J Cancer 119: 783-91.

9 Takahashi I, Emi Y, Kakeji Y et al. (2005) Increased antitumor activity in combined treatment TS-1 and docetaxel. A preclinical study using gastric cancer xenografts. Oncology 68: 130-7. 
10 Suto A, Kubota T, Fukushima M et al. (2006) Antitumor effect of combination of

S-1 and docetaxel on the human breast cancer xenograft transplanted into SCID mice. Oncol Rep 15: 1517-1522.

11 Yamaguchi K, Shimamura T, Hyodo I, et al. (2006) Phase I/II study of docetaxel and S-1 in patients with advanced gastric cancer. Br J Cancer 94: 1803-1808.

12 Gridelli C, Ardizzoni A, Ciardiello F et al. (2008) Second-Line Treatment of Advanced Non-small Cell Lung Cancer [State of the Art: Concise Review], J Thorac Oncol 3: 430-440.

13 Nelli F, Naso G, De Pasquale Ceratti A. et al. (2004) Weekly vinorelbine and docetaxel as second-line chemotherapy for pretreated non־small cell lung cancer patients: a phase I-II trial. J Chemother 16: 392-399.

14. Pectasides D, Kalofonos HP, Samantas E et al. (2001) An out-patient second-line chemotherapy with gemcitabine and vinorelbine in patients with non-small cell lung cancer previously treated with cisplatin-based chemotherapy. Anticancer Res 21: 3005-3010.

15. Spiridonidis CH, Laufman LR, Carman L et al. (2001) Second-line chemotherapy for non-small-cell lung cancer with monthly docetaxel and weekly gemcitabine: a phase II trial. Ann Oncol 12: 89-94. 
16. Takeda K, Negoro S, Tamura T et al. (2009) Phase III trial of docetaxel plus gemcitabine versus docetaxel in second-line treatment for non-small-cell lung cancer: results of a Japan Clinical Oncology Group trial (JCOG0104). Ann Oncol 20: $835-841$.

17 Hanna N, Shepherd FA, Fossella FV et al. (2004) Randomized phase III trial of pemetrexed versus docetaxel in patients with non-small-cell lung cancer previously treated with chemotherapy. J Clin Oncol 22: 1589-97.

18 Shepherd FA, Rodrigues Pereira J, Ciuleanu T, Hainsworth JD (2005) Erlotinib in Previously Treated Non-Small-Cell Lung Cancer. N Eng J Med 323: 123-32.

19 Spigel DR, Lin M, O'Neill V et al. (2008) Final survival and safety results from a multicenter, open-label, phase $3 \mathrm{~b}$ trial of erlotinib in patients with advanced non`small cell lung cancer. Cancer 112: 2749-55.

20 Kubota K, Nishiwaki Y, Tamura T et al. (2008) Efficacy and safety of erlotinib monotherapy for Japanese patients with advanced non-small cell lung cancer: a phase II study. J Thorac Oncol 3: 1439-45.

21 Ramlau R, Gervais R, Krzakowski M et al. (2006) Phase III study comparing oral topotecan to intravenous docetaxel in patients with pretreated advanced non־small-cell lung cancer. J Clin Oncol 24: 2800-7. 
22 Jones S, Thompson D, Barton J et al. (2008) A randomized phase II trial of oral topotecan versus docetaxel in the second-line treatment of non-small-cell lung cancer. Clin Lung Cancer 9: 154-9.

23 Takiguchi Y, Moriya T, Asaka-Amano Y et al. (2007) Phase II study of weekly irinotecan and cisplatin for refractory or recurrent non-small cell lung cancer. Lung Cancer 58: 253-9.

24 Chen YM, Perng RP, Tsai CM, Whang-Peng J (2006) A phase II trial of gemcitabine plus UFUR combination chemotherapy in non-small-cell lung cancer patients failing previous chemotherapy. Lung Cancer 52: 333-8.

25 Atagi S, Kawahara M, Kusunoki Y et al. (2008) Phase I/II study of docetaxel and S-1 in patients with previously treated non`small cell lung cancer. J Thorac Oncol 3: $1012-7$.

26 Ajani JA, Faust J, Ikeda K et al. (2005) Phase I pharmacokinetic study of S-1 plus cisplatin in patients with advanced gastric carcinoma. J Clin Oncol 23: 6957-65.

27 Yamamoto N, Tamura T, Kamiya Y et al. (2000) Correlation between docetaxel clearance and estimated cytochrome P450 activity by urinary metabolite of exogenous cortisol. J Clin Oncol 18: 2301-2308.

28 Sekine I, Yamamoto N, Nishio K, N Saijo N (2008) Emerging ethnic differences in 
lung cancer therapy. Br J Cancer 99: 1757-1762.

29 Park SR, Park MS, Park YL, et al. (2007) CYP2A6 genetic polymorphism as a predictive marker for clinical outcomes in patients with metastatic gastric carcinoma treated with S-1 plus docetaxel. 2007 ASCO Annual Meeting Proceedings. J Clin Oncol 25:230s. (abstr 4633). 
Table 1. Patient characteristics $(n=38)$

No. of patients $(\%)$

Median age

65 years; range, $44-74$ years

Gender

Male

Female

Histology

Adenocarcinoma

Squamous Cell Carcinoma

Others

Stage

IIIB

IV

Relapse after surgery

IIIBa $^{\mathrm{a}}$

IVa
$10(26 \%)$

$18(47 \%)$

$23(61 \%)$

$15(40 \%)$

$30(79 \%)$

$4(11 \%)$

$4(11 \%)$

$10(26 \%)$

$3(8 \%)$

7 (18\%)

ECOG PS 
Smoking history

Current/Former

Never

Number of previous chemotherapy regimens

1

2

Previous chemotherapy

Platinum-containing

Gefitinib
$35(92 \%)$

$24(63 \%)$

$14(37 \%)$

$23(61 \%)$

$15(39 \%)$

$7(18 \%)$

ECOG PS, Eastern Cooperative Oncology Group performance status.

aRestaging after relapse. 
Table 2. Overall response rates according to RECIST ( $n=38)$

\begin{tabular}{lrrrrl} 
CR & PR & SD & PD & NE & ORR \\
\hline & & & & & \\
0 & 7 & 25 & 6 & 0 & $18.4 \%(95 \%$ CI, $7.7-34.3 \%)$ \\
\hline
\end{tabular}

$R E C I S T$ response evaluation criteria in solid tumors, $C R$ complete response, $P R$ partial response, $S D$ stable disease, $P D$ progressive disease, $N E$ not evaluable, $O R R$ overall response rate, $C I$ confidence interval. 
Table 3. Adverse effects according to National Cancer Institute Common Terminology

Criteria for Adverse Events (version 3) $(n=38)$

Toxicity

All grades

Grade 3 or 4

No.

$(\%)$

No.

$(\%)$

Hematological toxicity

Neutropenia

Leukopenia

Anemia

Thrombocytopenia

Febrile neutropenia ${ }^{a}$

Gastrointestinal toxicity

Stomatitis

Nausea

Vomiting

Diarrhea

Metabolic/Laboratory
31

$(82 \%)$

$19 \quad(50 \%)$

22

$(58 \%)$

$8 \quad(21 \%)$

18

(47\%)

3

$(8 \%)$

0

$1 \quad(3 \%)$

1

$(3 \%)$

AST

15

$$
\text { (39\%) }
$$

0 
Hyperbilirubinemia

ALT

Hypercreatinemia

Other toxicity

Anorexia

Hand-foot skin reaction

Fatigue

Hyperpigmentation

Weight loss

Pneumonitis

10

$(26 \%)$

1

$(3 \%)$

$10 \quad(26 \%)$

0

3

$(8 \%)$

0



(


Legend for the figure

Figure 1: Kaplan-Meier survival curves demonstrating overall (solid line) and progression-free (dashed line) survival. OS overall survival, PFS progression-free survival 
Figure 1

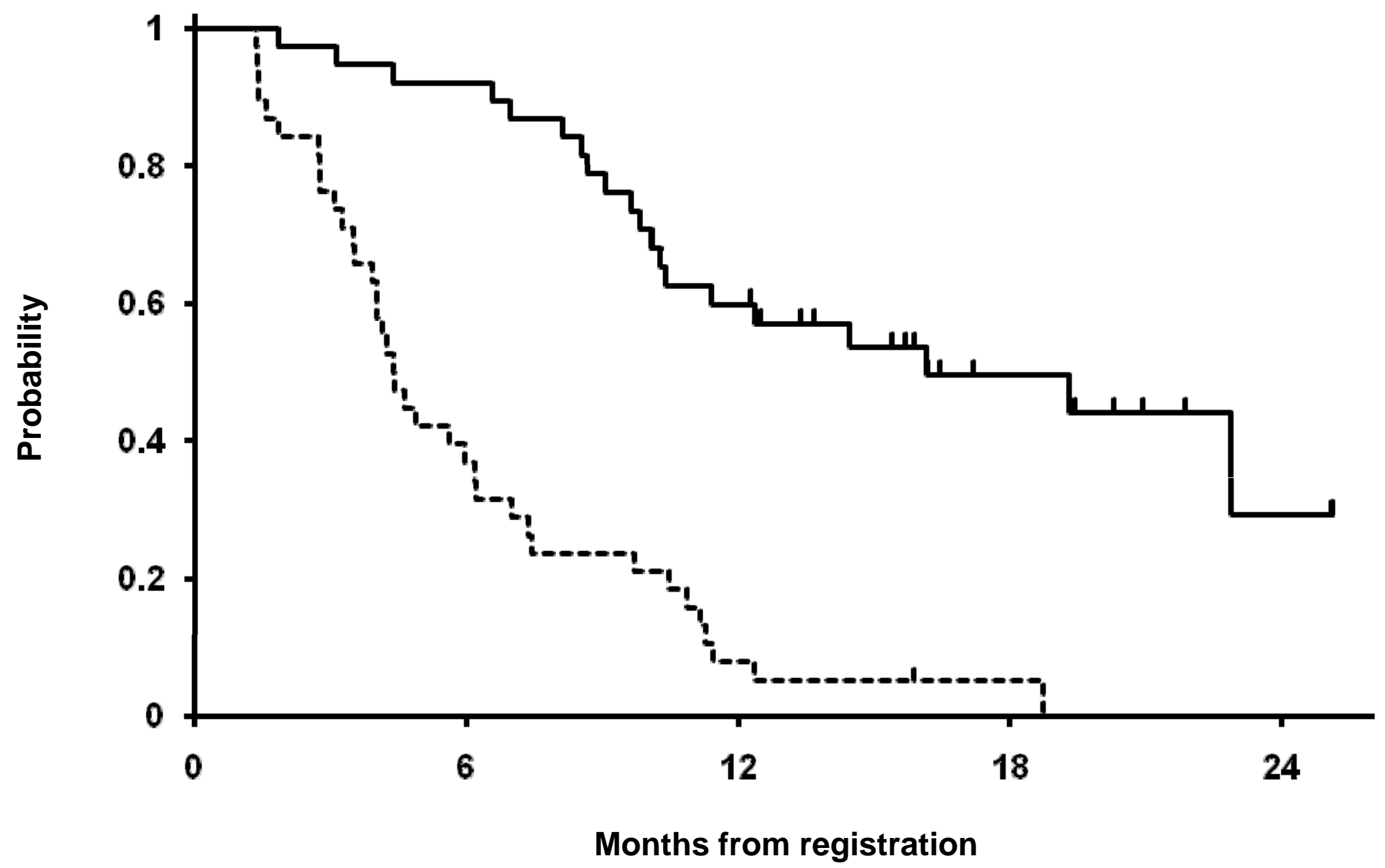

\# Patients at risk

\begin{tabular}{lrrrrr} 
Months & 0 & 6 & 12 & 18 & 24 \\
\hline OS & 38 & 35 & 22 & 9 & 2 \\
PFS & 38 & 14 & 3 & 1 & 0
\end{tabular}

